\title{
Actitud de futuros maestros frente al uso de TIC en educación: un análisis descriptivo ${ }^{1}$
}

\author{
Dra. Carolina Flores-Lueg ${ }^{2}$ \\ Universidad del Bío-Bío, Chile
}

\begin{abstract}
Resumen: Desde hace algún tiempo se han implementado una serie de políticas centradas en promover la incorporación de las TIC en los procesos formativos de todos los niveles educacionales y se ha invertido gran cantidad de recursos económicos en la dotación de la infraestructura e implementos vinculados a las tecnologías. No obstante a los esfuerzos realizados, el impacto que han tenido las tecnologías en los aprendizajes de los estudiantes no ha sido el esperado, y una de las razones responde específicamente a variables personales, entre ellas, la actitud que presenta el profesorado respecto a incorporar estas herramientas en el proceso de enseñanza y aprendizaje. Este trabajo da cuenta de los resultados más relevantes de una investigación descriptiva llevada a cabo en una universidad pública de Chile, donde participaron 175 estudiantes de Pedagogía, a quienes se les aplicó una escala de medición de actitud frente a las TIC en la educación, que buscaba precisamente detectar su apreciación personal respeto al uso de estas herramientas en los procesos educativos. Los resultados evidencian una actitud favorable de parte de los futuros maestros, lo que es coincidente con diversos estudios encontrados en la literatura que se centraron en medir esta variable.

Palabras clave: TIC, actitud, formación del profesorado, Educación Superior.

Abstract: Since a period of time, a series of policies focused on promoting the incorporation of ICT on the formative processes of all educational levels have been implemented and a great amount of economic resources have been invested in the endowment of infrastructure and implements linked to technology. However, despite the efforts, the impact that technologies have had on the students learning hasn't been the expected, and one of the reasons responds specifically to personal variables, among them, the attitude teachers show towards the incorporation of these tools in the teaching and learning process. This piece of work shows the most relevant results from a descriptive research, made in a public university in Chile, where 175 pedagogy students took part, and an attitude measuring scale towards ICT in education was applied to them, which precisely intended to detect their personal appreciation about the use of these tools in the educative processes. The results show a favorable attitude from the trainee teachers, coincident with many studies found in the literature focused on measuring this variable.
\end{abstract}

Keywords: ICT, attitude, training of teachers, Higher Education.

\section{Introducción}

La omnipresencia de las herramientas tecnológicas y digitales en la mayoría de los ámbitos sociales está generando escenarios altamente dinámicos, complejos e inciertos, lo que sin lugar a dudas presenta nuevos desafíos a las instituciones y a las personas. Dentro de este contexto, la educación adopta una relevancia sin precedentes, por cuanto en ella recae la responsabilidad de formar a los ciudadanos para el siglo XXI, quienes no sólo deben ser capaces de enfrentar los nuevos cambios y lograr las habilidades necesarias para hacer uso de las herramientas tecnológicas que van emergiendo, sino que, además, requieren desarrollar aquellas competencias que les permitan aprender durante toda la vida (lifelong learning) y participar activamente

\footnotetext{
${ }^{1}$ Este trabajo se enmarca dentro la tesis doctoral titulada «Factores que inciden en la valoración de estudiantes de Pedagogía sobre sus competencias digitales: El caso de la Universidad del Bío-Bío (Chile)», financiada por el Ministerio de Educación de Chile y la Universidad del Bío-Bío, a través del Proyecto MECESUP UBB:0704-2011.

2 Educadora de Párvulos (Maestra de Infantil), Magíster en Ciencias de la Educación, Máster en Educación y Tecnologías de la Información y la Comunicación, Doctora en Investigación Educativa por la Universidad de Alicante. Docente del Departamento de Ciencias de la Educación, Facultad de Educación y Humanidades, Universidad del Bío-Bío, Chile.
} 
dentro de este mundo cada vez más globalizado, asumiendo una actitud crítica y reflexiva basada en la interacción y la comunicación.

La realidad que estamos viviendo, mediada por las tecnologías digitales e Internet, ha impactado también en los diversos procesos que ocurren al interior de los centros educativos, pero claramente la labor que está desempeñando el profesorado en las aulas se constituye en una variable clave en lo que respecta a la generación de ambientes de aprendizaje enriquecidos, renovados y pertinentes al mundo tecnologizado que viven los niños y jóvenes. En este sentido, es importante destacar que desde hace algún tiempo en el ámbito de la educación se evidencia una creciente preocupación por implementar políticas y estrategias tendientes a promover la incorporación de las TIC en el proceso de enseñanza y aprendizaje, entre ellas, por ejemplo, se destaca una importante inversión económica centrada en dotar a los establecimientos educacionales de la infraestructura e implementos tecnológicos necesarios para apoyar los procesos formativos; se han diseñado y ejecutado proyectos de formación en TIC destinados a todos los miembros de la comunidad educativa (directivos, administrativos, profesorado, estudiantes e inclusive miembros de las familias); se han definido estándares en el uso de TIC, entre muchas otras acciones.

No obstante a todos los esfuerzos realizados por los gobiernos y diversas instituciones, el impacto que han tenido las tecnologías en los aprendizajes de los discentes no ha sido el esperado o ha sido más lento en comparación con otras áreas (González y de Pablos, 2015; Peirano y Domínguez, 2008; Valverde, Garrido y Fernández, 2010). Esta situación, como plantean Silva y Astudillo (2012), puede ser explicada a partir de las complejidades que presentan los procesos de innovación dentro del ámbito de la educación debido a ciertas barreras que van emergiendo, sobre todo en lo que respecta a la integración de las TIC como recursos de apoyo a la labor docente.

De acuerdo con algunos autores (Sancho Gil et al., 2008; Valverde et al.,2010), dentro de las barreras más comunes para integrar las herramientas tecnológicas en las practicas docentes, se encuentran las falta de tiempo, falta de confianza en el aporte de estos medios para la mejora de práctica docente y el aprendizaje del alumnado; falta de capacitación; prejuicios y predilecciones del profesorado respecto a las formas de uso y aplicación desde el punto de vista de los "apropiadas" o "inapropiadas" que pueden ser, entre muchas otras razones. Sumado a ello, existen diversos estudios (Kale \& Goh, 2014; Gargallo et al., 2006, 2010; Silva y Astudillo, 2012; Suárez-Rodríguez et al., 2012; Tejedor, García-Valcárcel y Prada, 2009; Trujillo, Núñez y Navío, 2011) donde se destaca que un factor clave para la integración de las tecnologías en el aula es la actitud que adopta el profesor, y también la de los estudiantes (García-Valcárcel, 2011), particularmente respecto a los computadores y a Internet. Al respecto, concordamos con Morales (2000), cuando señala que el impacto de la tecnología sólo puede esperarse, a nivel individual, cuando se realiza un cambio de actitud, acorde con el cambio social que implica la tecnología y que afecta al individuo en su contexto.

Cuando hablamos de actitud se puede decir que no es un constructo fácil de definir, ya que en la literatura disponible desde el ámbito de psicología existen múltiples perspectivas que buscan explicar la forma en cómo se estructuran y manifiestan las actitudes en las personas (Kerlinger \& Lee, 2002; Hogg, Vaughan \& Haro, 2010; Sánchez y Mesa, 1997). No obstante, se aprecia que en la mayoría de ellas se alude a un componente evaluativo frente a algún objeto de actitud que predispone a actuar de determinada manera. En consecuencia, la actitud hace alusión a experiencias internalizadas por los sujetos, asociadas a cierta organización de las creencias, reacciones o capacidades críticas (Eiser, 1989, citado en Sarabia, 1992,136). 
A partir de lo anterior, entendemos que la actitud corresponde al conjunto de creencias, pensamientos o predisposiciones que tiene una persona frente a una determinada situación u objeto, lo que le conduce a emitir juicios a favor o en contra, de agrado o desagrado, etc. y la condiciona para actuar de determinada manera. Al transferir este significado de actitud hacia las TIC (objeto de actitud), se puede decir entonces, que la actitud que presenta el profesorado frente a estas herramientas se va a entender como el conjunto de creencias, pensamientos o predisposiciones que tiene un docente frente a las TIC e Internet, lo que le lleva a emitir juicios a favor o en contra de estos recursos y lo condiciona para incorporarlas en sus prácticas pedagógicas (Flores-Lueg y Roig-Vila, 2016). Por consiguiente, el significado y sentido que le atribuye el profesorado a estas herramientas responde a factores netamente subjetivos, asociado a la forma en cómo va construyendo su acercamiento cognitivo, afectivo y conductual frente a los objetos tecnológicos, específicamente en lo referido a su uso e incorporación efectiva dentro de sus prácticas educativas.

Finalmente, destacamos que la actitud no es un constructo directamente observable, por lo que sólo puede medirse en forma indirecta y, como señalan Reich y Adcock (1980:31), la mayoría de las mediciones se basan en pruebas de reportes verbales y se concentran en gran parte en medir uno u otro de los componentes actitudinales: afectivos, cognitivos y conativos. Las manifestaciones verbales de las actitudes se denominan opiniones y expresan un posicionamiento evaluativo o predictivo de la persona respecto del objeto de su opinión (Sarabia, 1992:134).

En lo que respecta a la detección de la actitud que presentan estudiantes de pedagogía y el profesorado en ejercicio sobre las TIC aplicadas a la educación, diversos estudios (Álvarez et al., 2011; Domínguez, 2011; Tejedor y García-Valcárcel, 2006; Tejedor et al., 2009; López, 2011; Marín y Reche, 2012; Pérez Fernández y Vílchez, 2013; Pino y Soto, 2010; Puentes et al. 2013; Ramírez et al. , 2012; Sanabria y Hernández, 2011), evidencian una actitud positiva frente a la utilización de las TIC en el proceso educativo $\mathrm{y}$, también, una valoración positiva respecto a las potencialidades que estos recursos pueden ofrecer en favor del aprendizaje. No obstante a estos resultados, conviene destacar que también hay algunos estudios (Gutiérrez et al., 2010), que evidencian una actitud en muchos casos negativa de los estudiantes de Magisterio frente al uso de estas herramientas, además de un escaso conocimiento sobre su utilización, por lo que estos atores concluyen que los futuros maestros no está tan familiarizados con las TIC como se piensa, que incluso que ignoran su potencial didáctico y las posibles formas de integración en el currículum de la enseñanza obligatoria.

Dada la relevancia que adopta el componente actitudinal del profesorado para la integración real de las TIC en el proceso educativo, específicamente desde una perspectiva pedagógica, este estudio tuvo como propósito central detectar la actitud que poseen futuros docentes frente a la incorporación de las TIC en educación.

\section{Metodología}

Para dar respuesta al propósito de este estudio, se utilizó un diseño metodológico descriptivo, de carácter transversal o transaccional, por cuanto las variables fueron medidas en un solo momento.

El contexto de estudio correspondió a la Facultad de Educación y Humanidades de la Universidad del Bío-Bío, institución es de carácter Pública y Estatal, ubicada en la Región del Biobio de Chile. Los programas de pregrado que imparte la Facultad incluyen la formación de profesores para los niveles de Ed. Parvularia, Básica y Media, a través de las siguientes 9 carreras de Pedagogía: 
Pedagogía en Educación Parvularia, Pedagogía en Educación General Básica, Pedagogía en Educación Básica con Especialidad, Pedagogía en Castellano, Pedagogía en Inglés, Pedagogía en Educación Física, Pedagogía en Historia y Geografía, Pedagogía en Ciencias Naturales y Pedagogía en Educación Matemática.

\subsection{Muestra}

Para la selección de la muestra se ha considerado como técnica el muestreo no probabilístico, caracterizado por la intencionalidad del investigador, quien intenta buscar los casos que pueden proporcionar el máximo de información (Alaminos, 2006: 45). Dentro del muestreo no probabilístico se ha optado por el muestreo de subgrupos homogéneos, porque se ha seleccionado una muestra de estudiantes que comparten una situación común, siendo ésta la realización de la actividad de práctica profesional, ubicada curricularmente en su último año de formación. La muestra quedó conformada por 175 estudiantes ( $n=175)$, donde el $79.4 \%$ correspondió a mujeres y un $20.6 \%$ a varones (ver Figura 1). En cuanto a la edad, la media fue de prácticamente 24 años $(S D=2.51)$ (ver Figura 2).

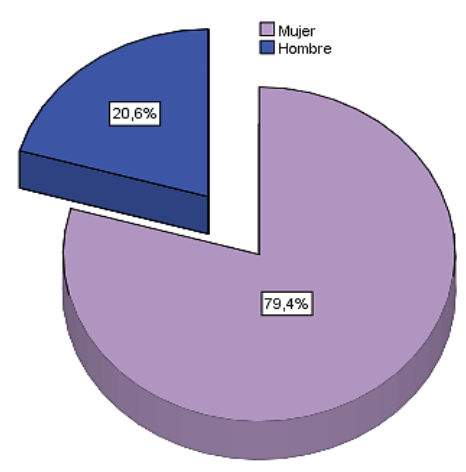

Figura 11. Distribución del sexo en la muestra total $(n=175)$

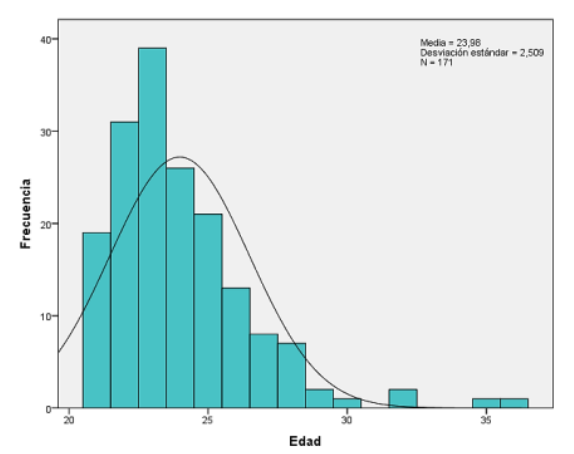

Figura 2. Distribución de la edad en la muestra $(n=171)$

Fuente: Elaboración propia mediante IBM SPSS Statistics 20

\subsection{Instrumentos}

Para la obtención de la información se seleccionó el instrumento denominado "Escala de Actitud de los profesores universitarios ante el uso de las tecnologías de la información y comunicación (TIC) en su práctica docente" (García-Valcárcel y Tejedor, 2007), cuyo coeficiente de consistencia interna alfa de Cronbach es .961. Desde el punto de vista de su estructura, esta escala está compuesta por 30 reactivos (50\% positivos y 50\% negativos), cuyas categorías de respuesta son: MD: Muy en desacuerdo (1), D: Desacuerdo (2), I: Indiferente, sin opinión elaborada (3), A: Acuerdo (4), MA: Muy de acuerdo (5). Si bien, originalmente fue construida con la finalidad de detectar la actitud de los profesores universitarios hacia la integración de las TIC en la docencia, al analizar detalladamente cada uno de los reactivos se consideró posible su adaptación para ser aplicada a estudiantes de las carreras de pedagogía que se encuentran en su última etapa de formación.

Cabe destacar que la versión adaptada de este instrumento fue sometida a un riguroso proceso de validación de contenido por medio del juicio de expertos y una validación de constructo a través de una aplicación piloto, donde se comprobó su validez mediante un Análisis Factorial Confirmatorio (AFC). El método empleado ha 
sido el de Máxima Verosimilitud, ya que el supuesto de normalidad multivariada no se cumplió totalmente debido a las asimetrías presentadas en algunos de los ítems. La Tabla 1 presenta el resumen de los estadísticos de ajuste utilizados y como se puede observar, el valor RMSEA se encuentra ligeramente por encima de .080 pero es altamente significativo para un $p<.001$ [IC (95\%) de entre: .085 y .101]; por tanto indica un ajuste aceptable. Por su parte, el resto de índices supera el corte de .800 o se acerca bastante a él. Aunque no se puede afirmar que el ajuste del modelo teórico con los datos empíricos sea muy elevado, sí se puede concluir que es suficientemente bueno como para que el resultado del AFC fuera fiable.

Tabla 1 AFC. Escala de actitud de estudiantes de Pedagogía frente a las TIC. Índices de bondad de ajuste

\begin{tabular}{ccccc}
\hline \multicolumn{1}{c}{ Modelo } & RMSEA & NFI & IFI & CFI \\
\hline Dos factores & $.093 * * *$ & .789 & .821 & .818 \\
\hline$* * *$ Significativo & con $p<000$ & & &
\end{tabular}

Por su parte, la Tabla 2 presenta los coeficientes ponderados estandarizados de los ítems en los 2 factores teóricos predefinidos. Todos los coeficientes son elevados, de manera que el resultado del AFC confirma la estructura dos factores, presentándose una leve correlación entre ambos.

Tabla 2. Análisis Factorial Confirmatorio: Escala de actitud frente a las TIC aplicadas a educación $(n=172)$

\begin{tabular}{|c|c|c|c|}
\hline Ítem & Enunciado & Fact. 1 & Fact. 2 \\
\hline ACT 1 & $\begin{array}{l}\text { El uso de las TIC favorece un aprendizaje activo } \\
\text { por parte de los alumnos/as. }\end{array}$ & .878 & \\
\hline ACT 2 & $\begin{array}{l}\text { Considero que son muy importantes las TIC para la } \\
\text { enseñanza en la actualidad. }\end{array}$ & .866 & \\
\hline ACT 4 & $\begin{array}{l}\text { Me parece positivo ir integrando progresivamente } \\
\text { las TIC en mi asignatura. }\end{array}$ & .861 & \\
\hline ACT 3 & $\begin{array}{l}\text { Los profesores/as debemos esforzarnos en } \\
\text { actualizarnos para aprovechar las posibilidades } \\
\text { didácticas de las TIC. }\end{array}$ & .845 & \\
\hline ACT 24 & $\begin{array}{l}\text { Me parece conveniente que haga un esfuerzo por } \\
\text { integrar las TIC en mis clases. }\end{array}$ & .786 & \\
\hline ACT 20 & $\begin{array}{l}\text { La utilización de las TIC en algunas actividades es } \\
\text { un buen modo de aprender para los alumnos/as. }\end{array}$ & .764 & \\
\hline ACT 8 & $\begin{array}{l}\text { Pienso que mis clases pueden mejorar si utilizo las } \\
\text { TIC. }\end{array}$ & .763 & \\
\hline ACT 5 & $\begin{array}{l}\text { Me encantaría trabajar en un centro que contara con } \\
\text { variados recursos tecnológicos. }\end{array}$ & .760 & \\
\hline ACT 13 & $\begin{array}{l}\text { Mi práctica docente puede enriquecerse gracias a } \\
\text { las posibilidades que me aportan las TIC. }\end{array}$ & .749 & \\
\hline ACT 11 & Debería introducir las TIC en mis clases. & .731 & \\
\hline ACT 17 & $\begin{array}{l}\text { Estoy dispuesto/a a aprender las posibilidades de } \\
\text { las TIC en la enseñanza. }\end{array}$ & .710 & \\
\hline ACT 9 & $\begin{array}{l}\text { Las TIC debería ser utilizadas por todos los } \\
\text { profesores/as en las distintas asignaturas. }\end{array}$ & .686 & \\
\hline ACT 25 & $\begin{array}{l}\text { Considero que el uso de las TIC ayuda al profesor/a } \\
\text { a realizar mejor su labor. }\end{array}$ & .682 & \\
\hline ACT 27 & $\begin{array}{l}\text { Considero que los profesores deberían utilizar las } \\
\text { TIC para facilitar el aprendizaje de los/as } \\
\text { estudiantes. }\end{array}$ & .676 & \\
\hline ACT 21 & $\begin{array}{l}\text { Las TIC me proporcionan flexibilidad de espacio y } \\
\text { tiempo pata comunicarme con mis alumnos. }\end{array}$ & .651 & \\
\hline
\end{tabular}


ACT 16 En el centro educativo donde realizo mi práctica deberían preocuparte por mejorar las infraestructuras actuales en TIC.

ACT 18 No me parece beneficioso introducir las TIC en la .527

inv. docencia.

ACT 19 Mis prácticas docentes no se van a mejorar por el

inv. uso de las TIC.

ACT 7 Considero que las TIC son entorpecedoras en el

inv. trabajo docente.

26 Siento que mis clases pierden eficacia si incorporo

inv. las TIC.
ACT 14 Tiene poco sentido creer que las TIC van a cambiar

inv. la docencia.

10 Considero que es irrelevante usar las TIC en la

$\begin{array}{ll}\text { inv. } & \text { docencia. } \\ \text { ACT } & 12 \mathrm{Mi} \text { labor como profesor/a no mejora por el uso de }\end{array}$

inv. las TIC.

ACT 6 Considero que las TIC sólo sirven para adornar la

inv. docencia.

23 Me agobia tanta información en Internet.

inv. la adquisición de algunas destrezas intelectuales básicas.

ACT 22 La utilización de las TIC no permite desarrollar un inv. aprendizaje significativo para los estudiantes. Correlación entre factores

Nota. Todos los coeficientes indicados son significativos con $p<.000$. Fuente. Elaboración propia.

Finalmente, a partir de los análisis realizados en el proceso de validación, la escala quedó conformada por 27 ítems que presentaron una fiabilidad elevada (Alfa de Cronbach $=0.863$, IC al 95\% de confianza: $.828-.894$ ), altamente significativo con $\mathrm{p}<.001$. La fiabilidad por dimensiones se puede observar en la Tabla 3.

Tabla 3. Análisis de fiabilidad. Escala de Actitud estudiantes Pedagogía hacia las TIC aplicadas en Educación $(n=136)$

\begin{tabular}{lccccc}
\hline Factor & $\mathrm{N}^{\mathrm{o}}$ ítems & $\begin{array}{c}\text { Alfa de } \\
\text { Cronbach }\end{array}$ & \multicolumn{2}{c}{ IC 95\% } & \multicolumn{2}{c}{ Anova } \\
\hline Dimensión 1 & 16 & .972 & $.964-.978$ & $\mathrm{~F}=12.48 ;$ & $\mathrm{p}=.000$ \\
Dimensión 2 & 11 & .920 & $.900-.938$ & $\mathrm{~F}=9.73 \quad ; \quad \mathrm{p}=.000$ \\
Escala completa & 27 & .863 & $.828-.894$ & $\mathrm{~F}=134.49 ;$ & $\mathrm{p}=.000$ \\
\hline
\end{tabular}

Fuente. Elaboración propia

En síntesis, se puede señalar que versión adaptada de la Escala "Actitud de los profesores universitarios ante el uso de las tecnologías de la información y comunicación (TIC) en su práctica docente" está formada por 2 dimensiones visiblemente separadas, que explican un $66,37 \%$ de la varianza. Asimismo, se observa que la fiabilidad es muy elevada, tanto en cada una de las dimensiones como en la escala completa, lo que también es coherente con la fiabilidad encontrada en la versión original. Por lo tanto, el instrumento empleado presenta validez estructural y fiabilidad.

\section{Resultados}

En primer lugar es importante destacar que se definieron dos variables, una por cada factor de la escala. Si bien, se debería emplear el método habitual de acumulación de puntos, es decir, que la puntuación de cada variable se obtiene en base al sumatorio de los valores numéricos asignados a cada ítem, la falta de respuestas en 
algunos de los ítems, recomienda que en su lugar se utilice el método de la media aritmética de las respuestas emitidas (valor que está también en escala 1 - 5: desacuerdo - acuerdo). Asimismo, para el Factor 2, previamente se ha invertido la escala de respuesta de los ítems, de manera que en ambas variables una puntuación más alta indique una postura más favorable frente a aquello que se está midiendo, vale decir, hacia las TIC aplicadas a educación.

Calculadas ambas variables más la puntuación total, se procede al análisis descriptivo y exploratorio de las mismas, basado en los índices de asimetría y curtosis y en el Test Kolmogorov-Smirnov de bondad de ajuste al modelo normal de la campana de Gauss (ver Tabla 4).

Tabla 4. Ajuste al modelo normal de la variable actitud frente a las TIC en educación

\begin{tabular}{lccc}
\hline & Factor 1 & Factor 2 & Punt. Total \\
\hline Asimetría & -1.262 & 0.821 & -0.168 \\
Curtosis & 2.171 & 0.171 & 2.922 \\
Test K-S: P-sig & $.007^{* *}$ & $.003^{* *}$ & $.005^{* *}$ \\
\hline
\end{tabular}

NS = no significativo ( $p>, 050)$ Buen ajuste

** Altamente Significativo al 1\% desvío grave

En el Factor 1 los índices de asimetría y curtosis indican una muy clara separación del modelo de la campana normal, sobre todo por una marcada asimetría hacia la derecha (ver Figura 3). De hecho el Test KS de bondad de ajuste presenta un desvío altamente significativo $(p<.01)$ con respecto al mismo. En el Factor 2, aunque los índices descriptivos indican un grado de asimetría leve y una altura dentro de la normalidad, también se encuentra un desvío estadísticamente significativo para $p<.01$ sobre la campana de Gauss (ver Figura 4). Finalmente en la puntuación total, se observa una buena simetría pero la altura (curtosis) es mucho mayor que la de campana normal (ver Figura 5) por lo que de nuevo el desvío es significativo con $p<.01$ sobre el modelo normal.

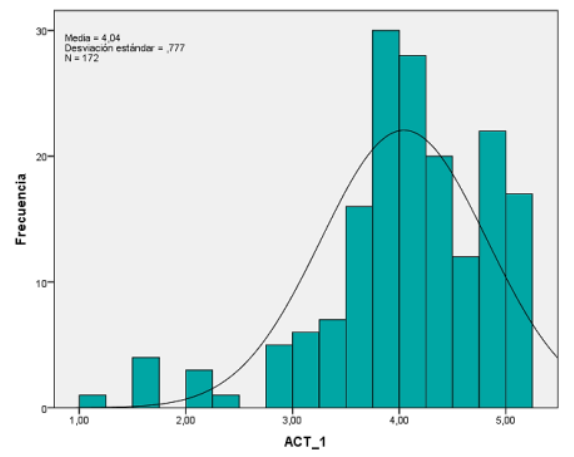

Figura 3. Histograma Factor 1. Escala de Actitud frente a las TIC. Fuente. Elaboración propia 


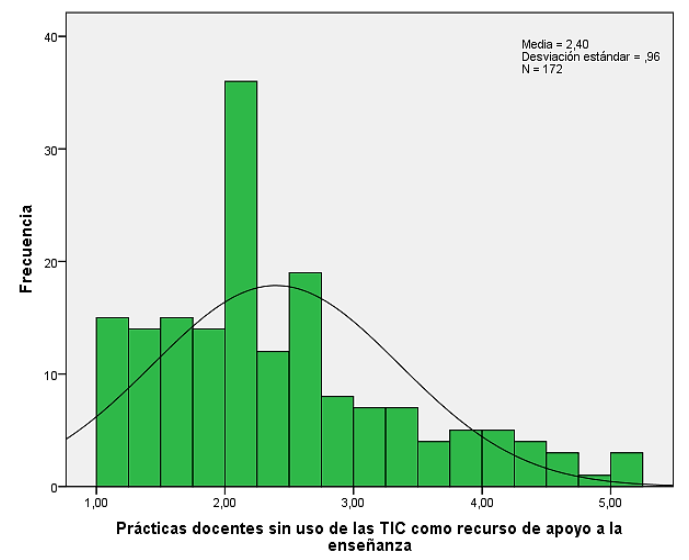

Figura 4. Histograma Factor 2. Escala de Actitud frente a las TIC. Fuente. Elaboración propia

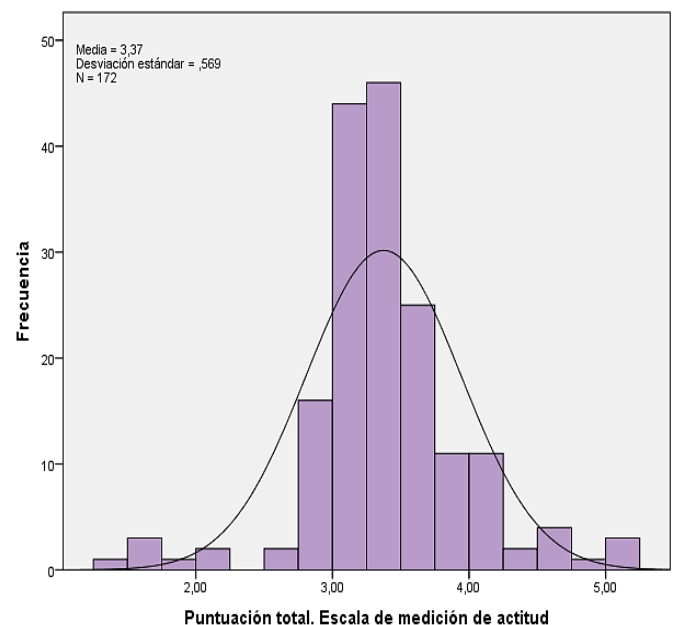

Figura 5. Histograma Puntuación total. Escala de Actitud frente a las TIC. Fuente. Elaboración propia

La falta de normalidad en las tres variables recomienda que los estadísticos más adecuados sean las medianas y los rangos semi-intercuartiles. Sin embargo, se puede apreciar que entre medias y medianas hay bastante similitud. Los valores obtenidos indican puntuaciones, en general elevadas en el Factor 1 y con bastante homogeneidad entre sujetos; mientras que en el Factor 2 el promedio es notablemente más bajo y, además, está acompañado de una mayor variabilidad entre estudiantes. Por su parte la puntuación total tiene una media/mediana centrada en la escala, acompañada de bastante homogeneidad entre sujetos. Los resultados descriptivos se pueden apreciar en la Tabla 5. 
Tabla 5. Análisis descriptivo. Variable

Actitud de los estudiantes de Pedagogía

frente a las TIC

\begin{tabular}{|c|c|c|c|}
\hline$N=172$ & Factor 1 & Factor 2 & Punt. Total \\
\hline Media & 4.04 & 2.40 & 3.37 \\
\hline IC 95\%: límite inferior & 3.93 & 2.25 & 3.29 \\
\hline $\begin{array}{l}\text { IC 95\%: límite } \\
\text { superior }\end{array}$ & 4.16 & 2.54 & 3.46 \\
\hline $\begin{array}{l}\text { Error típico de la } \\
\text { media }\end{array}$ & 0.06 & 0.07 & 0.04 \\
\hline Mediana & 4.06 & 2.18 & 3.33 \\
\hline Mínimo & 1.19 & 1.00 & 1.33 \\
\hline Máximo & 5.00 & 5.00 & 5.00 \\
\hline Desviación estándar & 0.78 & 0.96 & 0.57 \\
\hline Varianza & 0.60 & 0.92 & 0.32 \\
\hline $\begin{array}{l}\text { Rango } \\
\text { intercuartil }\end{array}$ & 0.47 & 0.55 & 0.23 \\
\hline $\begin{array}{l}\text { Coeficiente } \\
\text { Variación }\end{array}$ & 19.31 & 40.00 & 16.91 \\
\hline
\end{tabular}

Fuente. Elaboración propia

En la Tabla 6 y 7 se presenta el detalle de las frecuencias obtenidas por cada uno de los indicadores señalados en los factores que estructuran el instrumento empleado, a saber: Uso de las TIC en las prácticas docentes como apoyo didáctico para favorecer aprendizajes y Prácticas docentes sin uso de las TIC como recurso de apoyo a la enseñanza.

Tabla 6.Factor 1: Uso de las TIC en las prácticas docentes como apoyo didáctico para favorecer aprendizajes

\begin{tabular}{|c|c|c|c|c|c|c|c|c|}
\hline \multirow[b]{2}{*}{ Indicadores } & \multirow[b]{2}{*}{$n$} & \multirow[b]{2}{*}{$M$} & \multirow[b]{2}{*}{$S D$} & \multicolumn{5}{|c|}{ Frecuencia } \\
\hline & & & & $\begin{array}{r}\mathrm{MD} \\
(1)\end{array}$ & $\begin{array}{l}\mathrm{D} \\
(2)\end{array}$ & $\mathrm{I}(3)$ & $\mathrm{A}(4)$ & MA(5) \\
\hline $\begin{array}{l}\text { 2. Considero que son muy } \\
\text { importantes las TIC para la } \\
\text { enseñanza en la actualidad. } \\
\text { 1. El uso de las TIC } \\
\text { favorece un aprendizaje } \\
\text { activo por parte de los } \\
\text { alumnos/as. }\end{array}$ & 172 & 4.19 & 1.03 & 3.5 & 6.4 & 4.7 & 38.4 & 47.1 \\
\hline $\begin{array}{l}\text { 4. Me parece positivo ir } \\
\text { integrando progresivamente } \\
\text { las TIC en mi asignatura }\end{array}$ & 172 & 4.24 & 0.93 & 1.2 & 7.6 & 4.7 & 39.5 & 47.1 \\
\hline $\begin{array}{l}\text { 11. Debería introducir las } \\
\text { TIC en mis clases. }\end{array}$ & 171 & 4.09 & 0.95 & 1.2 & 8.2 & 9.4 & 42.7 & 38.6 \\
\hline $\begin{array}{l}\text { 3. Los profesores/as } \\
\text { debemos esforzarnos en } \\
\text { actualizarnos para } \\
\text { aprovechar las posibilidades } \\
\text { didácticas de las TIC. }\end{array}$ & 171 & 4.30 & 1.10 & 2.9 & 6.4 & 3.5 & 32.2 & 55.0 \\
\hline $\begin{array}{l}\text { 13. Mi práctica docente } \\
\text { puede enriquecerse gracias a } \\
\text { las posibilidades que me } \\
\text { aportan las TIC. }\end{array}$ & 172 & 4.09 & 0.98 & 2.3 & 7.0 & 9.3 & 42.4 & 39.0 \\
\hline
\end{tabular}


Tabla 6.Factor 1: Uso de las TIC en las prácticas docentes como apoyo didáctico para favorecer aprendizajes (Continuación)

\begin{tabular}{|c|c|c|c|c|c|c|c|c|}
\hline \multirow[b]{2}{*}{ Indicadores } & \multirow[b]{2}{*}{$n$} & \multirow[b]{2}{*}{$M$} & \multirow[b]{2}{*}{$S D$} & \multicolumn{5}{|c|}{ Frecuencia } \\
\hline & & & & $\begin{array}{c}\mathrm{MD} \\
(1)\end{array}$ & $\begin{array}{l}\mathrm{D} \\
(2)\end{array}$ & $\mathrm{I}(3)$ & $\mathrm{A}(4)$ & MA(5) \\
\hline $\begin{array}{l}\text { 25. Considero que el uso de las TIC ayuda } \\
\text { al profesor/a a realizar mejor su labor. }\end{array}$ & 171 & 3.86 & 1.10 & 4.1 & 9.9 & 13.5 & 40.9 & 31.6 \\
\hline $\begin{array}{l}\text { 24. Me parece conveniente que haga un } \\
\text { esfuerzo por integrar las TIC en mis clases. }\end{array}$ & 172 & 4.04 & 0.96 & 2.9 & 4.7 & 12.8 & 44.8 & 34.9 \\
\hline $\begin{array}{l}\text { 8. Pienso que mis clases pueden mejorar si } \\
\text { utilizo las TIC. }\end{array}$ & 171 & 4.00 & 1.03 & 2.3 & 9.4 & 10.5 & 41.5 & 36.3 \\
\hline $\begin{array}{l}\text { 27. Considero que los profesores deberían } \\
\text { utilizar las TIC para facilitar el aprendizaje } \\
\text { de los/as estudiantes. }\end{array}$ & 172 & 3.92 & 1.03 & 1.7 & 12.2 & 9.9 & 44.2 & 32.0 \\
\hline $\begin{array}{l}\text { 17. Estoy dispuesto/a a aprender las } \\
\text { posibilidades de las TIC en la enseñanza. }\end{array}$ & 171 & 4.12 & 1.01 & 4.1 & 4.1 & 8.2 & 43.3 & 40.4 \\
\hline $\begin{array}{l}\text { 20. La utilización de las TIC en algunas } \\
\text { actividades es un buen modo de aprender } \\
\text { para los alumnos/as. }\end{array}$ & 172 & 4.09 & 0.98 & 2.9 & 7.0 & 5.8 & 47.1 & 37.2 \\
\hline $\begin{array}{l}\text { 9. Las TIC debería ser utilizadas por todos } \\
\text { los profesores/as en las distintas } \\
\text { asignaturas. }\end{array}$ & 172 & 3.92 & 1.09 & 2.9 & 9.9 & 16.9 & 33.1 & 37.2 \\
\hline $\begin{array}{l}\text { 5. Me encantaría trabajar en un centro que } \\
\text { contara con variados recursos } \\
\text { tecnológicos. }\end{array}$ & 172 & 4.17 & 1.03 & 1.7 & 7.0 & 14.0 & 26.7 & 50.6 \\
\hline $\begin{array}{l}\text { 21. Las TIC me proporcionan flexibilidad } \\
\text { de espacio y tiempo pata comunicarme con } \\
\text { mis alumnos. }\end{array}$ & 172 & 3.94 & 0.99 & 1.7 & 8.1 & 17.4 & 40.1 & 32.6 \\
\hline $\begin{array}{l}\text { 16. En el centro educativo donde realizo } \\
\text { mi práctica deberían preocuparte por } \\
\text { mejorar las infraestructuras actuales en } \\
\text { TIC. }\end{array}$ & 170 & 3.51 & 1.15 & 6.5 & 10.6 & 31.8 & 27.6 & 23.5 \\
\hline
\end{tabular}

\section{Fuente. Elaboración propia}

Tabla 7. Factor 2: Prácticas docentes sin uso de las TIC como recurso de apoyo a la enseñanza

\begin{tabular}{|c|c|c|c|c|c|c|c|c|}
\hline \multirow{2}{*}{ Indicadores } & \multirow{2}{*}{$n$} & \multirow{2}{*}{$M$} & \multirow{2}{*}{$S D$} & \multicolumn{5}{|c|}{ Frecuencias } \\
\hline & & & & $\operatorname{MD}(1)$ & $\mathrm{D}(2)$ & $\mathrm{I}(3)$ & $\mathrm{A}(4)$ & MA(5) \\
\hline $\begin{array}{l}\text { 18. No me parece beneficioso introducir las } \\
\text { TIC en la docencia. }\end{array}$ & 168 & 2.11 & 1.24 & 39.3 & 35.7 & 7,7 & 9.5 & 7.7 \\
\hline $\begin{array}{l}\text { 19. Mis prácticas docentes no se van a } \\
\text { mejorar por el uso de las TIC. }\end{array}$ & 171 & 2.37 & 1.29 & 31.0 & 32.2 & 13.5 & 15.2 & 8.2 \\
\hline $\begin{array}{l}\text { 7. Considero que las TIC son entorpecedoras } \\
\text { en el trabajo docente. }\end{array}$ & 171 & 2.02 & 1.19 & 42.1 & 35.7 & 7.0 & 8.8 & 6.4 \\
\hline $\begin{array}{l}\text { 26. Siento que mis clases pierden eficacia si } \\
\text { incorporo las TIC. }\end{array}$ & 172 & 2.23 & 1.26 & 33.7 & 36.6 & 11.0 & 9.9 & 8.7 \\
\hline $\begin{array}{l}\text { 14. Tiene poco sentido creer que las TIC van } \\
\text { a cambiar la docencia. }\end{array}$ & 172 & 2.56 & 1.30 & 27.3 & 26.2 & 17.4 & 21.5 & 7.6 \\
\hline $\begin{array}{l}\text { 6. Considero que las TIC sólo sirven para } \\
\text { adornar la docencia. }\end{array}$ & 171 & 2.26 & 1.25 & 32.2 & 36.3 & 13.5 & 9.4 & 8.8 \\
\hline $\begin{array}{l}\text { 15. Las TIC no permiten a los alumnos/as } \\
\text { ejercitarse en la adquisición de algunas } \\
\text { destrezas intelectuales básicas. }\end{array}$ & 172 & 2.68 & 1.29 & 22.1 & 27.9 & 19.8 & 20.3 & 9.9 \\
\hline $\begin{array}{l}\text { 22. La utilización de las TIC no permite } \\
\text { desarrollar un aprendizaje significativo para } \\
\text { los estudiantes. }\end{array}$ & 172 & 2.65 & 1.40 & 25.0 & 32.6 & 9.9 & 18.0 & 14.5 \\
\hline 23. Me agobia tanta información en Internet. & 170 & 2.54 & 1.31 & 27.6 & 27.1 & 18.2 & 18.2 & 8.8 \\
\hline $\begin{array}{l}\text { 10. Considero que es irrelevante usar las TIC } \\
\text { en la docencia. }\end{array}$ & 172 & 2.22 & 1.27 & 36.0 & 33.1 & 11.6 & 11.0 & 8.1 \\
\hline $\begin{array}{l}\text { 12. Mi labor como profesor/a no mejora por } \\
\text { el uso de las TIC. }\end{array}$ & 171 & 2.71 & 1.40 & 24.6 & 26.9 & 16.4 & 17.0 & 15.2 \\
\hline
\end{tabular}

Fuente. Elaboración propia 


\section{Discusión y conclusiones}

Los resultados del análisis univariable muestran claramente una relación significativa y directa entre la valoración positiva de los estudiantes frente al uso de las TIC en las prácticas docentes como apoyo didáctico, resultados que coinciden con diversos estudios que se han centrado en detectar la actitud de estudiantes de Pedagogía y del profesorado en ejercicio sobre el uso de las TIC en educación (Álvarez et al., 2011; Domínguez, 2011; Fernández y Vílchez, 2013; González, 2011; Herrero, 2014; Karsenti y Lira, 2011; López, 2011; Marín y Reche, 2011; Morales et al. ,2015; Pérez y Vílchez, 2013; Pino y Soto, 2010; Puentes et al., 2013; Ramírez, et al. 2012; Salleh \& Laxman, 2014; Sanabria y Hernández, 2011; Sevillano y Fuero, 2013; Tejedor et al. , 2009), los que destacan, en términos generales, que tanto el profesorado en ejercicio como los estudiantes de formación inicial poseen una actitud favorable respecto a las herramientas tecnológicas incorporadas a educación.

La actitud favorable de los estudiantes también puede ser explicada principalmente porque que son alumnos jóvenes, mayoritariamente tienen entre $21 \mathrm{y}$ 24 años (68\%), y a lo largo de su vida han estado rodeados por las tecnologías, son usuarios activos de las mismas en su vida personal y, a la vez, han vivenciado los rápidos y permanentes avances de estas herramientas, por lo que no es de extrañar que su actitud frente a las TIC sea favorable (Rodríguez, 2000).

Si bien los resultados de este estudio son positivos, es importante tener en consideración que en la formación de los futuros maestros se aprecia la necesidad de abordar no solo el desarrollo de la competencia digital en todas sus dimensiones, sino que también enfatizar en el abordaje de variables personales y actitudinales respecto a las TIC, pues se requiere favorecer su capacidad de apertura y flexibilidad frente a estas herramientas incorporadas al proceso de enseñanza y aprendizaje dentro de escenarios altamente dinámicos y complejos, con la finalidad de que logren otorgarle valor educativo, que puedan aprovechar las diversas oportunidades de aprendizaje que ofrecen para que las integren con fines pedagógicos en sus prácticas docentes.

Por otra parte, consideramos que el abordaje adecuado de las variables personales, entre ellas, la positiva actitud que presentan los futuros maestros en la etapa de formación inicial, puede contribuir a aminorar tanto la brecha generacional entre el profesorado y los niños/jóvenes presentes en las aulas, como también puede ayudar a la creación de ambientes de aprendizaje enriquecidos e implementación de prácticas educativas pertinentes y contextualizadas y, por qué no decirlo, a la disminución de ciertas resistencias que presenta el profesorado en ejercicio a la hora de enfrentarse a las TIC.

En relación con lo anterior, concordamos con Díaz Barriga (2010), cuando señala que el uso dado por el profesorado a estas herramientas en el aula no ha impactado significativamente en la renovación de las prácticas docentes, pues, se ha traducido en hacer más eficiente lo que se ha venido haciendo tradicionalmente en las aulas (p.132). Asimismo, Cabero (2014) sostiene que existen factores relevantes en lo referido a la incorporación de las TIC a la práctica docente y que determinarán notablemente no solo si el docente llega a incorporar las TIC en su práctica educativa, sino también qué tecnologías utilizará y cuáles serán los sentidos de ello. Entre estos factores destaca la formación del profesorado, sus creencias respecto a la significación de estos instrumentos curriculares y el papel que puedan jugar en el contexto educativo actual, así como las actitudes existentes para determinar su movilización.

En consecuencia, si los programas de formación inicial del profesorado continúan enfatizando sólo en la adquisición de conocimientos de carácter técnico e 
instrumental respecto al uso de las TIC, se corre el riesgo de que las nuevas generaciones de profesores continúen reproduciendo los modelos tradicionales de enseñanza, independiente de si hacen uso de las TIC en el aula, pues, el sólo hecho de incorporarlas no necesariamente implica que se esté aprovechando todo el potencial educativo que estas herramientas aportan.

Finalmente, enfatizamos en que es necesario que se continúen desarrollando investigaciones en la línea de detectar aquellas variables personales del profesorado (actitudes, creencias, expectativas, entre otras) que caracterizan sus prácticas docentes con el uso de TIC, y aquellas que podrían estar interfiriendo en la incorporación efectiva de estas herramientas en el proceso de enseñanza y aprendizaje, con el fin de contar con información empírica que sirva de base para la elaboración de propuestas de intervención que contribuyan al desarrollo de buenas prácticas docentes con TIC.

\section{Referencias bibliográficas}

Alaminos, A. (2006). La dinámica de la interacción. En Alaminos y Castejón. Elaboración, análisis e interpretación de encuestas, cuestionarios y escalas de opinión (pp. 69-91).Alcoy: Editorial Marfil, S.A.

Álvarez, S. , Cuéllar, C. , López, B. , Adrada, C., Anguiano, R. , Bueno, A. , Comas , I. y Gómez, S., (2011). Actitudes de los profesores ante la integración de las TIC en la práctica docente. Estudio de un grupo de la Universidad de Valladolid. EDUTEC, Revista Electrónica de Tecnología Educativa, 35. Recuperado de http://goo.gl/h73dw

Cabero, J. (2014). La formación del profesorado en TIC: unas referencias conceptuales. En Silva y Salinas (Coords.). Innovando con TIC en la formación inicial docente. Aspectos teóricos y casos concretos, (pp. 175- 194). Santiago de

Chile: Universidad de Santiago de Chile. Recuperado de http://tecnologiaedu.us.es/tecnoedu/images/stories/ticfid_2014.pdf

Díaz Barriga, F. (2010). Integración de las TIC en el currículo y la enseñanza para promover la calidad educativa y la innovación. Pensamiento Iberoamericano, 7, 129-149. Recuperado de http://www.red-redial.net/referencia-bibliografica$\underline{54829 . \mathrm{html}}$

Domínguez, R. (2011). Formación, competencia y actitudes sobre las TIC del profesorado de secundaria: Un instrumento de evaluación. Etic@net, IX(10). Recuperado de http://www.ugr.es/ sevimeco/revistaeticanet/index.htm

Fernández, F. y Vílchez, J. (2013). Percepción de futuros maestros sobre el potencial de las TIC en la educación: de las expectativas a la realidad. Revista Fuentes, 13, 155-172. Recuperado de http://goo.gl/d7lpDP 
Flores-Lueg, C. y Roig-Vila, R. (2016). La actitud del profesorado: una variable a medir en el contexto de la integración educativa de las TIC. En Gómez Galán, J., López Meneses, E. y Molina García, L. (Eds). Instructional Strategies in Teacher Training, (pp. 110-130). UMET Press.

García-Valcárcel, A. y Tejedor, F. (2007). Estudio de las actitudes del profesorado universitario hacia la integración de las TIC en su práctica docente. Recuperado de http://www.utn.edu.ar/aprobedutec07/docs/123.doc

García-Valcárcel, A.(2011). Integración de las TIC en la docencia universitaria. España: Netbiblo S.L.

Gargallo, B., Suarez, J., y Almerich, G. (2006).La influencia de las actitudes de los profesores en el uso de las nuevas tecnologías. Revista Española de Pedagogía, 233, 45-66. Recuperado de https://dialnet.unirioja.es/servlet/articulo?codigo=1973265

Gargallo, B; Suárez, J.; Belloch, C. y Almerich, G. (2010). Perfiles actitudinales de los profesores ante las TIC e incidencia de las actitudes sobre su uso. Recuperado de http://goo.gl/RlWl1K

González Rodero, L. (2011). Las competencias TIC del alumnado. En GarcíaValcárcel (coord.). Integración de las TIC en la docencia Universitaria (pp. 7597). España: NETBIBLO, S.L.

González Pérez, A.; de Pablos, J. (2015). Factores que dificultan la integración de las TIC en las aulas. Revista de Investigación Educativa, 33 (2), 401-417. doi: http://dx.doi.org/10.6018/rie.33.2.198161

Gutiérrez Martín, A. , Palacios, A. y Torrego, L. (2010). La formación de los futuros maestros y la integración de las TIC en la educación: anatomía de un desencuentro. Revista de Educación, 352. Recuperado de http://www.revistaeducacion.educacion.es/re352/re352_TIC.pdf.

Herrero, R. (2014). El papel de las TIC en el aula universitaria para la formación en competencias del alumnado. Píxel-Bit. Revista de Medios y Educación, 45, 173188. doi: http://dx.doi.org/10.12795/pixelbit.2014.i45.12

Hogg, M., Vaughan, G. \& Haro, M. (2010). Psicología Social/ Social Psychology. Madrid: Editorial Médica Panamericana.

Kale, U. \& Goh, G. (2014). Teaching style, ICT experience and teachers' attitudes toward teaching with Web 2.0. Educ Inf Technol, 19, 41-60. doi: 10.1007/s10639-012-9210-3 
Kerlinger, F. y Lee, H. (2002). Investigación del comportamiento: Métodos de investigación en ciencias sociales ( $4^{\mathrm{a}}$ Ed.). México, DF.: McGraw Hill/Interamericana.

Karsenti, T. y Lira, M. L. (2011). ¿Están listos los futuros profesores para integrar las TIC en el contexto escolar?. El caso de los profesores en Quebec, Canadá. Revista Electrónica de Investigación Educativa, 13(1). Recuperado de http://redie.uabc.mx/vol13no1/contenido-karsentilira.html

López, A. (2011). Usos y actitudes de estudiantes universitarios futuros profesores sobre Tecnologías de la Información y la Comunicación (tic) y recursos sociales de internet. Questions. Revista Especializada de Periodismo y Comunicación, 1(31). Recuperado de http://goo.gl/NgKxN

Marín, V. y Reche, E. (2011). La Alfabetización digital del alumnado que accede a la Universidad de Córdoba. Edutec-e. Revista Electrónica de Tecnología Educativa, 35. Recuperado de http://edutec.rediris.es/Revelec2/Revelec35/pdf/Edutece_n35_Marin_Reche.pdf

Morales, C. (Coord.) (2000). Actitudes de los estudiantes y los docentes hacia la computadora y los medios de aprendizaje. México: ILCE. Recuperado de http://observatorio.ilce.edu.mx/documentos/Introduccion_actitudes.pdf

Morales, M., Trujillo, J.M. y Raso, F. (2015). Percepciones acerca de la integración de las TIC en el proceso de enseñanza-aprendizaje de la Universidad. Píxel-Bit. Revista de Medios y Educación, 46, 103-117. doi: http://dx.doi.org/10.12795/pixelbit.2015.i46.0

Peirano, C. y Domínguez, M.P. (2008). Competencia en TIC. El mayor desafío para la evaluación y el entrenamiento docente en Chile. Revista Iberoamericana de Evaluación Educativa, 1 (2), 106-123. Recuperado de http://www.rinace.net/riee/numeros/vol1-num2/art7.pdf

Pérez Fernández, F. y Vílchez, J. (2013). Percepción de Futuros Maestros sobre el potencial de las Tic en la Educación: de las Expectativas a la Realidad. Revista Fuentes, 13, 155-172. Recuperado de http://institucional.us.es/fuentes/gestor/apartados revista/pdf/campo/fazwddpk. pdf

Pino, M. y Soto, J. (2010). Identificación del dominio de competencias digitales en el alumnado de grado de magisterio. Teoría de la Educación. Educación y Cultura en la Sociedad de la Información (TESI), 11(3), 336-362. Recuperado de 
http://campus.usal.es/ revistas trabajo/index.php/revistatesi/article/view/7466/7 $\underline{483}$

Puentes, A., Roig, R., Sanhueza, S. y Friz, M. (2013). Concepciones sobre las Tecnologías de la Información y la Comunicación (TIC) y sus implicaciones educativas: Un estudio exploratorio con profesorado de la provincia de Nuble, Chile. Revista Iberoamericana de Ciencia, Tecnología y Sociedad, 8(22). Recuperado de http://rua.ua.es/dspace/bitstream/10045/35183/1/2013_Puentes_etal_CTS.pdf

Ramírez, E., Cañedo, I. y Clemente, M. (2012). Las actitudes y creencias de los profesores de secundaria sobre el uso de Internet en el aula. Comunicar, Revista Científica de Educomunicación, XIX (38), 147-155. doi: 10.3916/38-2012-0306

Reich, B. \& Adcock, C. (1980). Values, Attitudes \& Behaviour Change. Valores, actitudes y cambios de conducta. Traducción de Levesque. H. México: Compañía Editorial Continental, S.A.

Rodríguez, F. (2000). Las actitudes del profesorado hacia la informática. Pixel-Bit.

Revista de medios y Educación, 15, 91-103.Recuperado de http://www.sav.us.es/pixelbit/pixelbit/articulos/n15/n15art/art158.htm

Salleh, S. \& Laxman, K . (2014). Investigating the factors influencing teachers' use of ICT in teaching in Bruneian secondary schools. Educ Inf Technol, 19, 747762. doi:10.1007/s10639-013-9251-2

Sanabria, A. y Hernández, C. (2011). Percepción de los estudiantes y profesores sobre uso de las TIC en los procesos de cambio e innovación en la enseñanza superior. Aloma. Revista de Psicología, Ciències de l'Educació i de l'Esport, 29 , 273-290. Recuperado de http://www.revistaaloma.net/index.php/aloma/article/view/106/82

Sánchez, S. y Mesa, C (1997). Actitudes hacia la tolerancia y la cooperación en ambientes multiculturales. Evaluación y propuestas de intervención educativa. Recuperado de http://goo.gl/PDG4D5

Sancho, J.M., Ornellas, A., Sánchez, J.A. , Alonso, C. y Bosco, A. (2008). La formación del profesorado en el uso de las TIC: una aproximación desde la política educativa. Revista Praxis Educativa, XII(12),10-22. Recuperado de http://www.biblioteca.unlpam.edu.ar/pubpdf/praxis/n12a02sancho.pdf 
Sarabia, B. (1992). El aprendizaje y enseñanza de las actitudes. En Coll, C., Pozo, J. I., Sarabia, B. y Valls, E. Los contenidos de la Reforma. Enseñanza de conceptos, procedimientos y actitudes (pp. 133-198). Ediciones Santillana S.A.

Sevillano, M.C. y Fuero (2013). Formación Inicial del Profesorado en TICS: Un Análisis de Castilla- La Mancha. Profesorado. Revista de Currículum y Formación del Profesorado, 17(3), 151-183. Recuperado de http://www.ugr.es/ recfpro/rev173ART10.pdf

Silva, J. y Astudillo, A. (2007). Evaluación de la implementación y valoración de los estándares TIC para la formación inicial docente. Innovación Educativa, 7(41), 1-24. Recuperado de http://www.redalyc.org/pdf/1794/179421215005.pdf

Suárez-Rodríguez, J., M., Almerich, G., Díaz-García, I. y Fernández-Piqueras, R. (2012). Competencias del profesorado en las TIC. Influencia de factores personales y contextuales. Universitas Psychologica, 11(1), 293-309. Recuperado de http://revistas.javeriana.edu.co/index.php/revPsycho/article/viewFile/997/1537

Tejedor, F.J. y García-Valcárcel, A.(2006). Competencias de los profesores para el uso de las TIC en la enseñanza. Análisis de sus conocimientos y actitudes. Revista Española de Pedagogía, 64(233), 21-44. Recuperado de http://goo.gl/2bLaO

Tejedor, F.J., García-Valcárcel, A. y Prada, S. (2009). Medida de actitudes del profesorado universitario hacia la integración de las TIC. Comunicar, XVII(33), 115-124. doi:10.3916/c33-2009-03-002

Trujillo Torres, J.M. , López Núñez, J.A. y Pérez Navío, E. (2011). Caracterización de la Alfabetización digital desde la perspectiva del profesorado: la competencia docente digital. Revista Iberoamericana de Educación, 55(4). Organización de Estados Iberoamericanos para la Educación, Ciencia y la Cultura (OEI). Recuperado de http://www.rieoei.org/deloslectores/3879Trujillo.pdf

Valverde, J. , Garrido, M.C. y Fernández, R. (2010). Enseñar y aprender con tecnologías: Un modelo teórico para las Buenas Prácticas con TIC. TESI, 11 (3), 203-229. Recuperado de http://gredos.usal.es/jspui/bitstream/10366/72834/1/ENSENAR_Y_APRENDE R_CON_TECNOLOGIAS_UN_MO.pdf 